\title{
Aberrantly expressed long noncoding RNAs and genes in Parkinson's disease
}

This article was published in the following Dove Press journal:

Neuropsychiatric Disease and Treatment

\author{
Yong Zhou \\ Chengzhi Gu \\ Jia Li \\ Lianhai Zhu \\ Guoxiang Huang \\ Jie Dai \\ Huaiyu Huang \\ Department of Neurology, \\ The Second Affiliated Hospital of \\ Nantong University, The First People's \\ Hospital of Nantong, Nantong, China
}

Correspondence: Huaiyu Huang

Department of Neurology, The Second Affiliated Hospital of Nantong University,

The First People's Hospital of Nantong,

No 6 Northern Haierxiang Road,

Chongchuan District, Nantong 22600I, Jiangsu, China

Tel +86 I3 809087798

Fax +8605 I38 506 I0I2

Email huanghuaiyu99@163.com
Purpose: Parkinson's disease (PD) is a common neurodegenerative movement disorder, but the pathogenesis remains elusive. This study was aimed to explore key genes and long noncoding RNAs (lncRNAs) associated with PD.

Materials and methods: Three patients with PD and three normal controls were enrolled in the present study from July 12, 2017, to August 29, 2017. RNA sequencing and bioinformatics analysis were performed to obtain differentially expressed micro RNAs (DEmRNAs) and lncRNAs (DElncRNAs) between patients with PD and normal controls. PD-specific protein-protein interaction networks were constructed. DEmRNAs transcribed within a $100 \mathrm{~kb}$ window upstream or downstream of DElncRNAs were searched, which were defined as cis nearby targeted DEmRNAs of DElncRNAs. Datasets GSE57475 and GSE68719 were downloaded from the Gene Expression Omnibus database, which were used to validate the expression of selected DEmRNAs.

Results: A total of 857 DEmRNAs and 77 DElncRNAs were obtained between PD and normal controls. Natural killer cell-mediated cytotoxicity was a significantly enriched pathway in PD. ERBB2, HSPB1, and MYC were three hub proteins of PD-specific protein-protein interaction network. LOC105378701-TAL1, LOC102724104-CX3CR1, LOC105375056-TREML1/ TREML4, LOC105379392-ANK1, and LOC101928100-KLRK1/KLRD1 interactions were identified DElncRNA nearby targeted DEmRNA pairs in PD. Gene expression results validated by GSE57475 and GSE68719 were consistent with our RNA-sequencing results, generally.

Conclusion: This present study identified key genes and lncRNAs associated with PD, which will provide new clues for exploring the pathogenesis and developing potential biomarkers of PD.

Keywords: RNA-sequencing, mRNA, bioinformatics analysis, protein-protein interaction network

\section{Introduction}

As a common neurodegenerative movement disorder, Parkinson's disease (PD) is characterized by slowness of movement, rigidity, postural instability, and resting tremor. ${ }^{1}$ These clinical manifestations were resulted from progressive loss of dopamine producing neurons in the substantia nigra pars compacta and widespread intracellular aggregation of the protein alpha-synuclein, the principal component of the pathological hallmark of PD, Lewy bodies. ${ }^{2}$ Despite the increasing efforts for exploring the etiology of PD, the exact pathology of PD was not fully defined.

Long noncoding RNAs (lncRNAs) are non-protein-coding transcripts over 200 nucleotides bases long. Recently, accumulated evidences have emphasized the importance of lncRNAs in brain function and central nervous system (CNS) disorders. ${ }^{2-5}$ The functions of lncRNAs span from regulating brain evolution and neural development ${ }^{3}$ to mediating behavioral and cognitive processes. ${ }^{4,5}$ Moreover, lncRNAs were reported to regulate the transcription of nearby genes with cis-regulatory effects. Up to date, 
several lncRNAs such as NEAT1, SNHG1, MAPT-AS1, and HOTAIR have been demonstrated to play roles in PD. ${ }^{6-9}$ Yanxia Fan et al reported differentially expressed genes, ZFAND4, SRMS, UBL4B, PVALB, DIRAS1, PDP2, LRCH1, and MYL4 were potential biomarkers associated with progression rate of PD. ${ }^{10}$ The study of Jieshan Chi et al suggested that five significantly down-regulated mRNAs (MAPK8, CDC42, NDUFS1, COX4I1, and SDHC) and three significantly down-regulated miRNAs (miR-126-5p, miR19-3p, and miR-29a-3p), were potentially useful diagnostic markers in clinic. ${ }^{11}$

In this present study, we identified the differentially expressed lncRNAs (DElncRNAs) and mRNAs (DEmRNAs) between PD and normal controls by RNA sequencing and bioinformatics analysis. Identification of cis nearby targeted DEmRNAs of DElncRNAs and functional annotation of DEmRNAs would facilitate the exploration of the biological functions of DElncRNAs in PD. This study will provide new clues for understanding the pathogenesis and developing potential biomarkers of PD.

\section{Materials and methods}

\section{Patients and samples}

Three patients with PD and three normal controls were enrolled in the present study from July 12, 2017, to August 29, 2017. PD was diagnosed based on MDS clinical diagnostic criteria. ${ }^{12}$ Patients with other long-term chronic disease and serious disease were excluded. The details of these patients were as follows: a 54-year-old male with 3 years of slow movement and jitter of left upper limb at Hoehn-Yahr stage 1.5 ; a 60 -year-old male with $>4$ years of slow movement and progressive hand shaking at Hoehn-Yahr stage 2.5, and a 52-year-old male with $>2$ years of slow movement and hand shaking at Hoehn-Yahr stage 2.0, respectively. All these patients have no family history of PD. Three normal controls were 65-, 54-, and 53-year old healthy males. All individuals provided signed informed consent for use of their samples in this present study. The present study has been approved by the Ethics Committee of the First People's Hospital of Nantong.

From each participant, a $2.5 \mathrm{~mL}$ peripheral whole blood was collected in PAXgene ${ }^{\circledR}$ RNA blood tubes (PreAnalytiX $\mathrm{GmbH}$, Hombrechtikon, Switzerland) and stored at $-80^{\circ} \mathrm{C}$ prior to processing.

\section{RNA isolation and sequencing}

With PAXgene blood RNA kit (PreAnalytiX GmbH, Hombrechtikon, Switzerland), RNA isolation was conducted on the manufacturer's protocol. By using Nanodrop ND-2000 spectrophotometer (Thermo Fisher Scientific, Inc., Waltham, MA, USA), the concentration and purity of RNA were assessed. The integrity of RNA was assessed via a $2 \%$ agarose gel. Agilent 2100 bioanalyzer was used to obtain the RIN values. The criteria for cDNA library construction were as follows: 1) Total RNA $>5 \mu \mathrm{g}$; 2) concentration of RNA $\geq 200 \mathrm{ng} / \mathrm{mL}$; 3 ) OD 260/280 value $1.8-2.2$.

Ribosomal RNA was removed with Ribo-Zero Magnetic kit (EpiCentre, Madison, WI, USA). Then, RNA was purified and fragmented into fragments with 140-160 nt. The first cDNA strand was synthesized via RNA fragments primed with random hexamer primers. The second cDNA strand was synthesized with dUTP instead of dTTP. End repair was conducted by using End Repair Enzyme mix (NEB, Ipswich, MA, USA). Subsequently, 3' end adenylation and adapter ligation were performed. After digesting the second cDNA strand with UNG enzyme (Illumina, Inc., San Diego, CA, USA), PCR was performed for 15 cycles to amplify the libraries. Purification and recovery of libraries were performed by using commercial magnetic beads. Sequencing was performed on the Illumina Hiseq X-ten platform (Illumina, Inc.).

\section{Quality control of raw sequence and mapping of clean reads}

By using Base Calling version 0.11.4 (http://www.bioinformatics.babraham.ac.uk/projects/fastqc/), ${ }^{35}$ the FASTQ sequence data were obtained from the RNA-sequencing data. Reads with low quality (adaptor sequences, sequences with a quality score $<20$, and sequences with an $\mathrm{N}$ base rate of raw reads $>10 \%$ ) were removed with Cutadapt version 1.9.1 (https://cutadapt.readthedocs.io/en/stable/) ${ }^{36}$ to obtain the clean reads.

\section{Identification of DEmRNAs and DElncRNAs in PD compared with normal controls}

TopHat release 2.2.1 (http://tophat.cbcb.umd.edu//) $)^{37}$ was used to align the clean reads with the human reference genome, Ensemble GRCh38.p7 (ftp://ftp.ncbi.nlm.nih.gov/ genomes/Homo sapiens). ${ }^{38}$ With Cuffquant version 2.2.1 (http://cufflinks.cbcb.umd.edu/), ${ }^{39}$ expressions of mRNAs and lncRNAs were normalized and outputted. Fragments per Kilobase of exon per million fragments mapped (FPKM) was used to determine the transcription abundance of lncRNAs and mRNAs. With Cuffdiff version 2.2.1 (http://cufflinks. cbcb.umd.edu/), ${ }^{39}$ FPKM of IncRNAs and mRNAs were 
calculated. Both DEmRNAs and DElncRNAs were obtained with DESeq2 (http://bioconductor.org/packages/DESeq2/4) ${ }^{40}$ in R version 3.3.3 with $P$-value $<0.05$. Hierarchical clustering analyses of DElncRNAs and DEmRNAs were conducted by using R package "pheatmap".

\section{Functional annotation of DEmRNAs between PD and normal control}

Functional annotation, including Gene Ontology (GO) function and Kyoto Encyclopedia of Genes and Genomes (KEGG) pathway enrichment analyses of the DEmRNAs between PD and normal control, was performed using the GeneCoDis3 tool (http://genecodis.cnb.csic.es/analysis). ${ }^{41}$ False discovery rate (FDR) $<0.05$ was set as the cutoff for significance.

\section{Protein-protein interaction (PPI) network construction}

With the Biological General Repository for Interaction Datasets (BioGrid, http://www.uniprot.org/database/DB-0184), ${ }^{42}$ top 100 up- and downregulated DEmRNAs were scanned. PPI network was then constructed using Cytoscape software (version 3.3.0, http://www.cytoscape.org) ) $^{43}$ in order to further explore the biological functions of the DEmRNAs.

\section{Cis nearby targeted DEmRNAs of the DElncRNAs}

To obtain the targeted DEmRNAs of DElncRNAs with cis-regulatory effects, DEmRNAs transcribed within a $100 \mathrm{~kb}$ window upstream or downstream of DElncRNAs were searched, which were defined as cis nearby targeted DEmRNAs of DElncRNAs.

\section{Validation in the GEO dataset}

GSE57475 and GSE68719 datasets were downloaded from the Gene Expression Omnibus (GEO; https:/www.ncbi. nlm.nih.gov/geo/),${ }^{44}$ which consisted of 49 patients with PD and 93 normal controls, and 29 patients with PD and 44 normal controls, respectively. The dataset GSE57475 examined the blood sample and GSE68719 examined brain tissue, which were all from USA. The expression patterns of selected DEmRNAs were validated with GSE57475 and GSE68719 datasets.

\section{Results \\ RNA-sequencing data}

Total RNA isolated from each blood sample met the criteria for cDNA library construction and RNA sequencing. After trimming of the raw reads, $6.7 \times 10^{7}$ clean reads were obtained from each blood sample from patients with PD and normal controls. The mapping ratio was calculated following clean reads of each sample aligned to the human reference genome (GRCh38.p7). Mapping ratio of each sample was $>84 \%$.

\section{DEmRNAs and DElncRNAs between PD and normal controls}

A total of 857 DEmRNAs (304 upregulated and 553 downregulated DEmRNAs) and 77 DElncRNAs (38 upregulated and 39 downregulated DElncRNAs) between PD and normal controls were identified. The top ten up- and downregulated DElncRNAs and DEmRNAs between PD and normal controls are summarized in Tables 1 and 2, respectively. Hierarchical clustering analysis of DElncRNAs and top 100 DEmRNAs is displayed in Figure 1A and B, respectively. Furthermore, all these DElncRNAs were distributed in all chromosomes (chr.), with the exception of chr.15 and chr.16, and DEmRNAs were widely distributed in all chromosomes (Figure 1C).

\section{Functional annotation}

Blood coagulation (FDR $=4.11 \mathrm{E}-11)$, platelet activation $(\mathrm{FDR}=2.55 \mathrm{E}-09)$, plasma membrane $(\mathrm{FDR}=1.48 \mathrm{E}-11)$,

Table I Top ten up- and downregulated DElncRNAs between $\mathrm{PD}$ and normal controls

\begin{tabular}{|c|c|c|c|}
\hline DEIncRNAs & $\begin{array}{l}\text { Log2 fold } \\
\text { change }\end{array}$ & $P$-value & Regulation \\
\hline TM4SFI9-TCTEXID2 & $1.91 E+00$ & $7.4 \mathrm{IE}-07$ & Up \\
\hline LOCI01927369 & $1.21 \mathrm{E}+00$ & I.37E-04 & Up \\
\hline LOCI02724I04 & $1.04 \mathrm{E}+00$ & $1.60 \mathrm{E}-04$ & $U_{p}$ \\
\hline LINC0I87I & I.IIE+00 & I.79E-04 & $U_{p}$ \\
\hline LOCI05373420 & $1.25 \mathrm{E}+00$ & $9.26 \mathrm{E}-04$ & $U_{p}$ \\
\hline LOCI0537I464 & 7.73E-0I & $1.94 \mathrm{E}-03$ & $U_{p}$ \\
\hline LINC00943 & $9.86 \mathrm{E}-0 \mathrm{I}$ & $3.50 \mathrm{E}-03$ & $U_{p}$ \\
\hline LOCI05370060 & $1.09 \mathrm{E}+00$ & $4.4 \mathrm{IE}-03$ & Up \\
\hline LOC 101927012 & $9.74 \mathrm{E}-0 \mathrm{I}$ & $5.26 \mathrm{E}-03$ & $U_{p}$ \\
\hline LOC 105372055 & $9.94 \mathrm{E}-0 \mathrm{I}$ & $5.88 \mathrm{E}-03$ & Up \\
\hline LOC 102724765 & $-1.07 E+00$ & I.38E-04 & Down \\
\hline LOCI05369772 & $-1.34 \mathrm{E}+00$ & $4.76 \mathrm{E}-04$ & Down \\
\hline KRT73-ASI & $-1.25 \mathrm{E}+00$ & $5.00 \mathrm{E}-04$ & Down \\
\hline LOCI05379392 & $-9.44 \mathrm{E}-0 \mathrm{I}$ & $6.04 \mathrm{E}-04$ & Down \\
\hline JHDMID-ASI & $-8.08 \mathrm{E}-0 \mathrm{I}$ & $1.96 \mathrm{E}-03$ & Down \\
\hline LOCI05372185 & $-1.03 E+00$ & $2.02 \mathrm{E}-03$ & Down \\
\hline LOCI05377225 & $-7.0 \mathrm{IE}-0 \mathrm{I}$ & $2.02 \mathrm{E}-03$ & Down \\
\hline LOCI0537870I & $-1.18 \mathrm{E}+00$ & $2.15 \mathrm{E}-03$ & Down \\
\hline LOCI05375056 & $-1.12 \mathrm{E}+00$ & $2.34 \mathrm{E}-03$ & Down \\
\hline LOCI05373204 & $-7.02 \mathrm{E}-0 \mathrm{I}$ & $3.74 \mathrm{E}-03$ & Down \\
\hline
\end{tabular}

Abbreviations: DElncRNAs, differentially expressed long noncoding RNAs; PD, Parkinson's disease. 
Table 2 Top ten up- and downregulated DEmRNAs between PD and normal controls

\begin{tabular}{|c|c|c|c|}
\hline DEmRNAs & $\begin{array}{l}\text { Log2 fold } \\
\text { change }\end{array}$ & $P$-value & Regulation \\
\hline NINL & $1.67 \mathrm{E}+00$ & $6.23 \mathrm{E}-13$ & Up \\
\hline GZMB & $1.28 \mathrm{E}+00$ & $6.70 \mathrm{E}-11$ & Up \\
\hline COL6A2 & $1.23 \mathrm{E}+00$ & $1.48 \mathrm{E}-09$ & Up \\
\hline GZMH & I. $19 \mathrm{E}+00$ & 4.64E-09 & Up \\
\hline ERBB2 & $1.12 \mathrm{E}+00$ & $5.98 \mathrm{E}-09$ & $U_{p}$ \\
\hline FGFBP2 & $1.13 E+00$ & I.7IE-08 & $U_{p}$ \\
\hline GNLY & I. $10 \mathrm{E}+00$ & $6.61 \mathrm{E}-08$ & Up \\
\hline Clorf2I & $9.67 \mathrm{E}-0 \mathrm{I}$ & $7.48 \mathrm{E}-08$ & $U_{p}$ \\
\hline APOBEC $3 B$ & $1.23 \mathrm{E}+00$ & $9.63 \mathrm{E}-08$ & Up \\
\hline PRSS23 & $1.09 \mathrm{E}+00$ & I.07E-07 & Up \\
\hline LRRN3 & $-1.49 \mathrm{E}+00$ & $4.27 \mathrm{E}-14$ & Down \\
\hline KRT73 & $-1.72 \mathrm{E}+00$ & I.IIE-13 & Down \\
\hline MYL4 & $-1.29 \mathrm{E}+00$ & $3.20 \mathrm{E}-13$ & Down \\
\hline FKBP8 & $-1.05 \mathrm{E}+00$ & $5.03 \mathrm{E}-1 \mathrm{I}$ & Down \\
\hline KIAAI 324 & $-1.43 \mathrm{E}+00$ & $3.18 \mathrm{E}-10$ & Down \\
\hline$X K$ & $-1.08 \mathrm{E}+00$ & $2.8 I E-08$ & Down \\
\hline GNGII & $-1.13 E+00$ & $3.12 \mathrm{E}-08$ & Down \\
\hline TMEMI58 & $-1.19 \mathrm{E}+00$ & 4. $10 \mathrm{E}-07$ & Down \\
\hline IL6ST & $-6.84 \mathrm{E}-0 \mathrm{I}$ & $5.64 \mathrm{E}-07$ & Down \\
\hline LOCI02723750 & $-9.8|E-0|$ & $8.30 \mathrm{E}-07$ & Down \\
\hline
\end{tabular}

Abbreviations: DEmRNAs, differentially expressed micro RNAs; PD, Parkinson's disease.

protein binding (FDR $=2.40 \mathrm{E}-30)$, and cytoplasm (FDR $=2.54 \mathrm{E}-36)$ are significantly enriched $\mathrm{GO}$ terms in PD (Figure 2A-C). Natural killer cell-mediated cytotoxicity $(\mathrm{FDR}=2.36 \mathrm{E}-08)$, pathways in cancer $(\mathrm{FDR}=6.82 \mathrm{E}-06)$, and protein processing in endoplasmic reticulum $(\mathrm{FDR}=1.54 \mathrm{E}-05)$ are three significantly enriched KEGG pathways in PD (Figure 2D).

\section{PPI network}

The PD-specific PPI network was consisted of 186 nodes and 194 edges. ERBB2 (degree =13), HSPB1 (degree =13), and MYC (degree $=11$ ) were three hub proteins of PDspecific PPI network (Figure 3).

\section{Cis nearby targeted DEmRNAs of DElncRNAs}

A total of 39 DElncRNAs nearby targeted DEmRNA pairs were obtained which was consisted of 28 DElncRNAs and 36 DEmRNAs. LOC105378701-TAL1, LOC102724104CX3CR1, LOC105375056-TREML1/TREML4, LOC105379392-ANK1, and LOC101928100-KLRK1/ KLRD1 interactions were identified DElncRNAs nearby targeted DEmRNA pairs in PD (Table 3).

\section{Validation in the GEO dataset}

The expression patterns of six DEmRNAs including TAL bHLH transcription factor 1 (TAL1), triggering receptor expressed on myeloid cells like 1 (TREML1), triggering receptor expressed on myeloid cells like 4 (TREML4), erb-b2 receptor tyrosine kinase 2 (ERBB2), chemokine $(\mathrm{C}-\mathrm{X} 3-\mathrm{C})$ receptor $1(C X 3 C R 1)$ and ankyrin $1(A N K 1)$ were verified using GSE57475 dataset. As shown in Figure 4, TAL1, TREML1, TREML4, and ANK1 were downregulated while $E R B B 2$ and $C X 3 C R 1$ were upregulated in PD compared with normal controls, which were consistent with our RNAsequencing results (Figure 4).

In GSE68719 dataset, the expression patterns of ERBB2, $C X 3 C R 1$, and $A N K 1$ were similar to that in GSE57475, while for TAL1 and TREML1, it displayed the opposite. TREML4 was not found in GSE68719. The result may be due to the difference of the tissue types between GSE57475 and GSE68719. We further determined the expression of killer cell lectin like receptor D1 (KLRD1), a DEmRNA between PD and normal controls, and found that its expression status was consistent with our RNA-sequencing results (Figure 5).

\section{Discussion}

Increasing evidences have indicated that IncRNAs play important roles in the pathogenesis of PD. ${ }^{6-9}$ This present study identified abundant lncRNAs that were differentially expressed between PD and normal controls.

JHDM1D antisense 1 (JHDM1D-AS1) was an lncRNA that arises from the antisense strand of JHDM1D and was downregulated in PD in this present study. In in vitro and in vivo experiments, JHDM1D-AS1 was found to be upregulated in cancer cells and tumor tissues under nutrient starvation, which promotes tumorigenesis by upregulating angiogenesis and triggering inflammation. ${ }^{13}$ Accumulated evidence indicated that neuroinflammation plays key roles in the pathogenesis of neurodegenerative diseases. ${ }^{14,15}$ Angiogenesis has been found in various neurodegenerative diseases such as Alzheimer's disease (AD) ${ }^{16}$ and PD $^{1}$ which was speculated to make a contribution for neuroinflammation by failing to protect the parenchyma from peripheral immune cells and inflammatory or toxic factors in the peripheral circulation. ${ }^{1}$ Hence, we made a hypothesis that JHDM1D-AS1 might be involved with the process of PD by regulating angiogenesis and neuroinflammation. Further experiments are needed to explore the precise role of JHDM1D-AS1 in PD.

However, the biological functions of most identified DElncRNAs between PD and normal control remain unclear. 
A

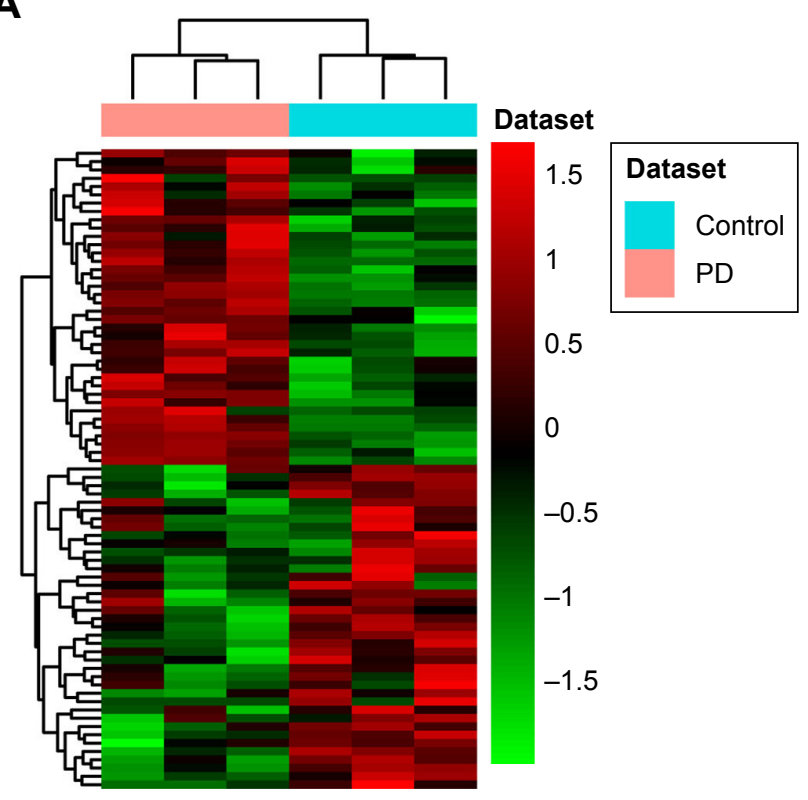

B

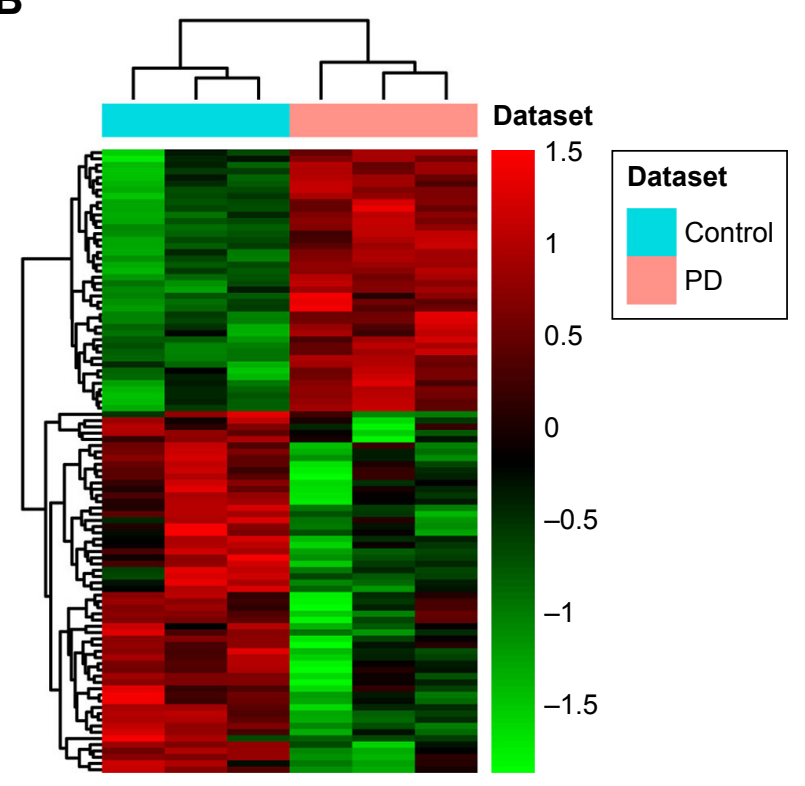

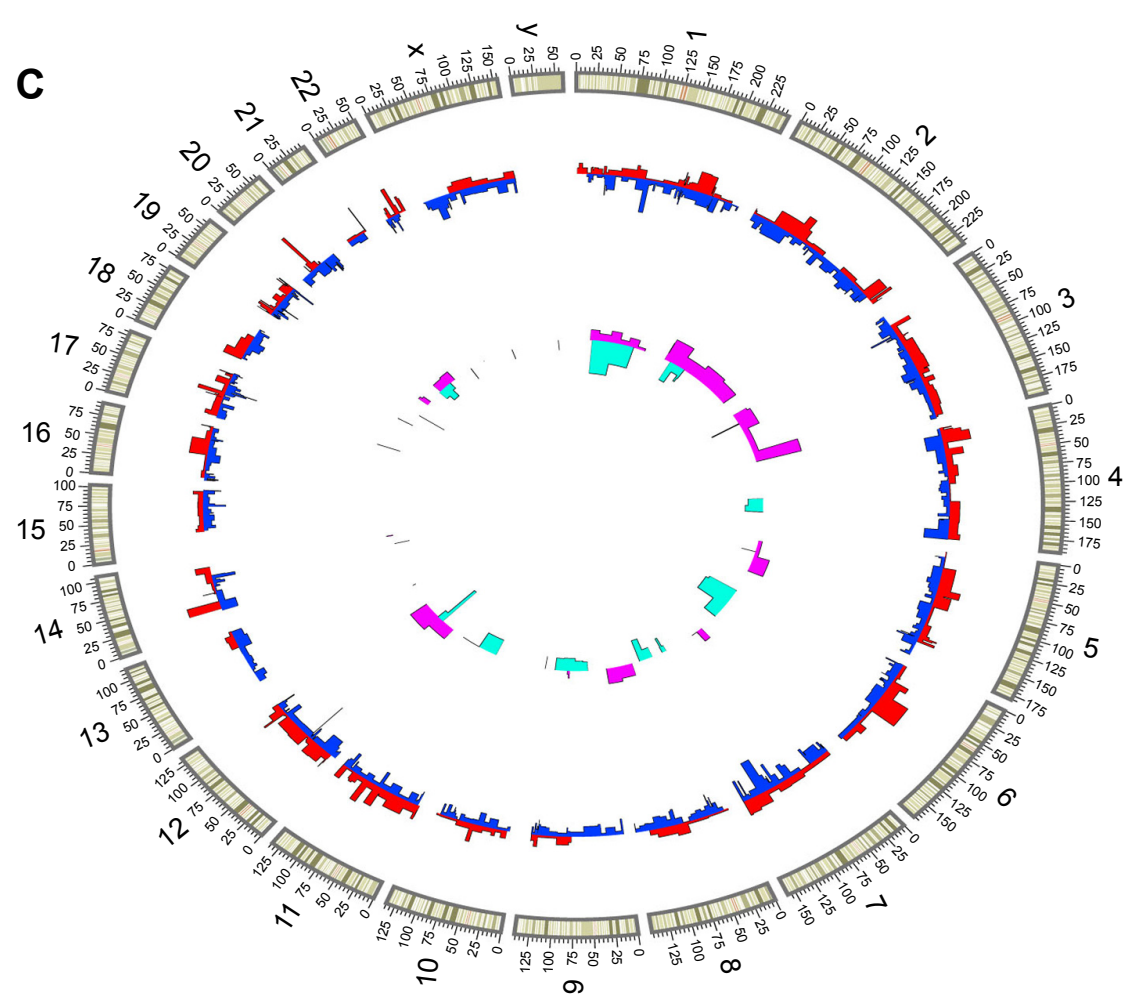

Figure I DElncRNAs and DEmRNAs between PD and normal controls.

Notes: (A, B) Hierarchical clustering results of DElncRNAs and top 100 DEmRNAs between PD and normal controls, respectively. Row and column represent DElncRNAs/ DEmRNAs and tissue samples, respectively. The color scale represents the expression levels. (C) Distribution of DElncRNAs and DEmRNAs on chromosomes. The outer layer cycle was the chromosome map of the human genome hgl9 (GRCh37). The red and blue inner layer represents the distribution of up- and downregulated DEmRNAs on different chromosomes, respectively. The pink and light blue inner layer represents the distribution of up- and downregulated DElncRNAs on different chromosome, respectively.

Abbreviations: DElncRNAs, differentially expressed long noncoding RNAs; DEmRNAs, differentially expressed micro RNAs; PD, Parkinson's disease.

Previous studies indicated that lncRNAs might regulate the expression of their nearby genes by cis-regulatory effects. ${ }^{17}$ To further research the functions of DElncRNAs in PD, we searched the DEmRNAs transcribed within a $100 \mathrm{~kb}$ window upstream or downstream of DElncRNAs which served as cis nearby targeted DEmRNAs of DElncRNAs.

In this present study, downregulated TAL1 was found in PD compared with normal controls, and interacted with 


\section{A}

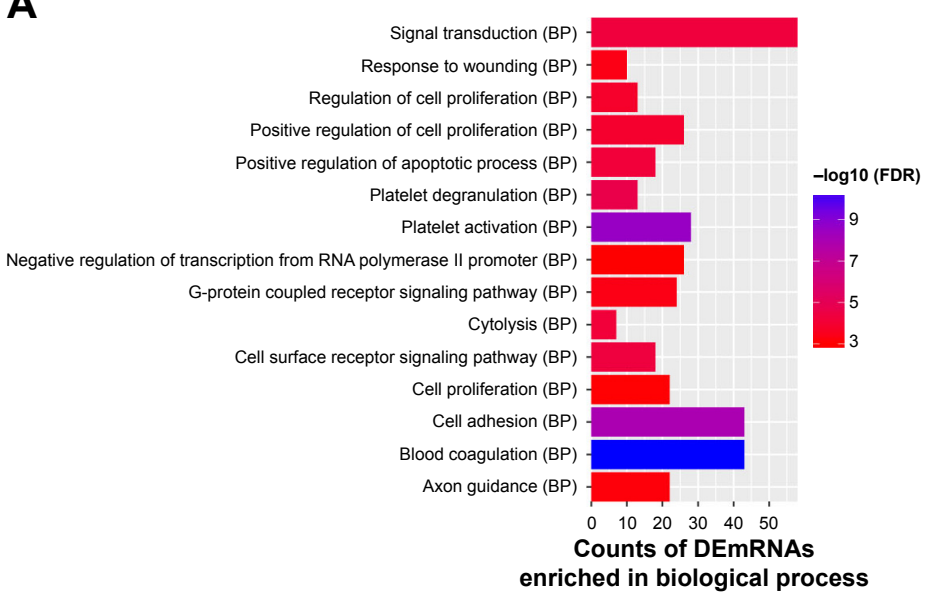

C

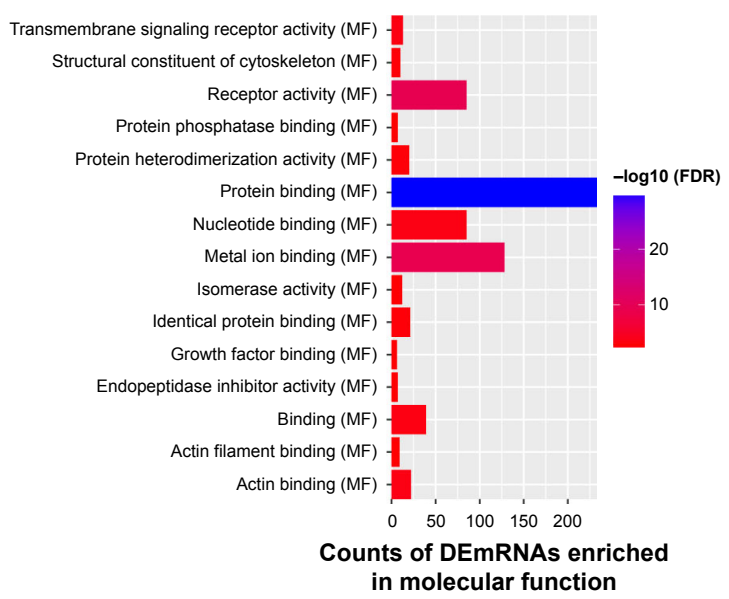

B

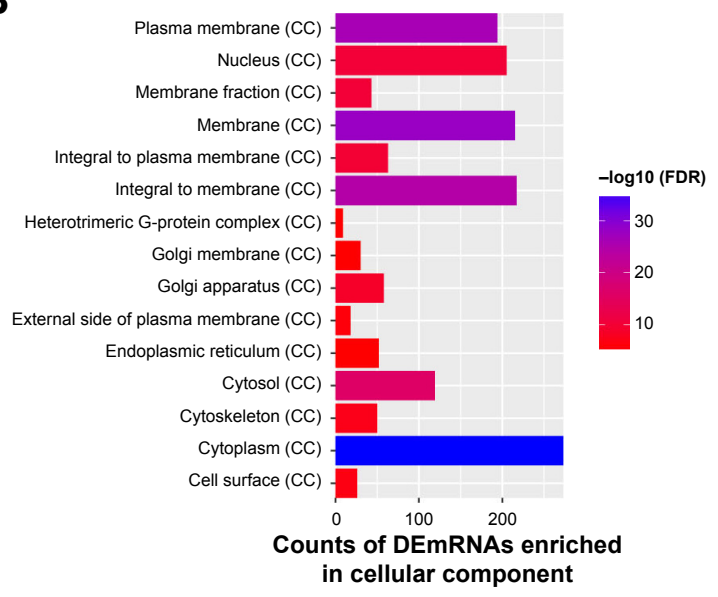

D

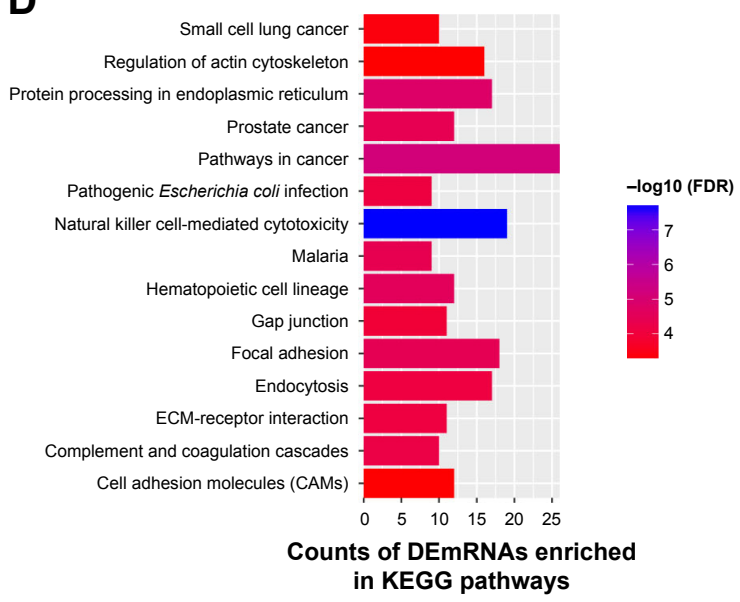

Figure 2 Significantly enriched GO terms and KEGG pathways of DEmRNAs between PD and normal controls.

Notes: (A) BP, (B) CC, (C) MF, and (D) KEGG pathways. The x-axis shows counts of DEmRNAs enriched in GO terms or KEGG pathways and the $y$-axis shows GO terms or KEGG pathways. The color scale represented -log FDR.

Abbreviations: BP, biological process; CC, cellular component; DEmRNAs, differentially expressed mRNAs; FDR, false discovery rate; GO, gene ontology; KEGG, Kyoto Encyclopedia of Genes and Genomes; MF, molecular function.

seven DEmRNAs of PD. Microglia is essential for innate neuroimmune function and CNS homeostasis, and plays crucial roles in neurodegeneration and brain aging. ${ }^{18}$ TAL1 is a transcription factor that involves with microglial aging. ${ }^{19}$ Hence, TAL1 was speculated to play key roles in initiation of PD by regulating many key DEmRNAs between PD and normal controls. ${ }^{20}$ Additionally, TAL1 was identified to be a nearby targeted DEmRNA of LOC105378701, which suggested that LOC105378701 might involve with PD by regulating the expression of TAL1 with cis effect.

CX3CR 1 is a specific receptor of fractalkine (also called $C X 3 C L 1$ ) that is exclusively expressed in microglia in the $\mathrm{CNS}^{21}$ Fractalkine/CX3CR 1 signaling plays an inhibitory role in control of microglial inflammatory response. $^{22}$ Knockout of $C X 3 C R 1$ was found to exacerbate inflammation and neurodegeneration in a 1-methyl-4phenyl-1,2,3,6-tetrahydropyridine neurotoxin model of PD. ${ }^{23}$ Sun et al demonstrated for the first time that $C X 3 C R 1$ is involved in the neuroinflammatory process in 1-methyl-4phenylpyridiniumion (MPP+) rat model of PD. ${ }^{24}$ Both the RNA-sequencing results and our gene expression validation results found that $C X 3 C R 1$ was upregulated in PD compared with normal control, which emphasized the importance of $\mathrm{CX} 3 \mathrm{CR} 1$ in PD. Further research is needed to explore its precise role in PD. Moreover, $C X 3 C R 1$ was a nearby targeted gene of LOC102724104, which suggested that LOC102724104 might be a potential regulator of $\mathrm{PD}$ by regulating $C X 3 C R 1$.

$E R B B 2$, also known as human epidermal growth factor receptor 2 (HER2), a receptor tyrosine kinase, was originally identified based on its role in cancer research. Wang et al first 


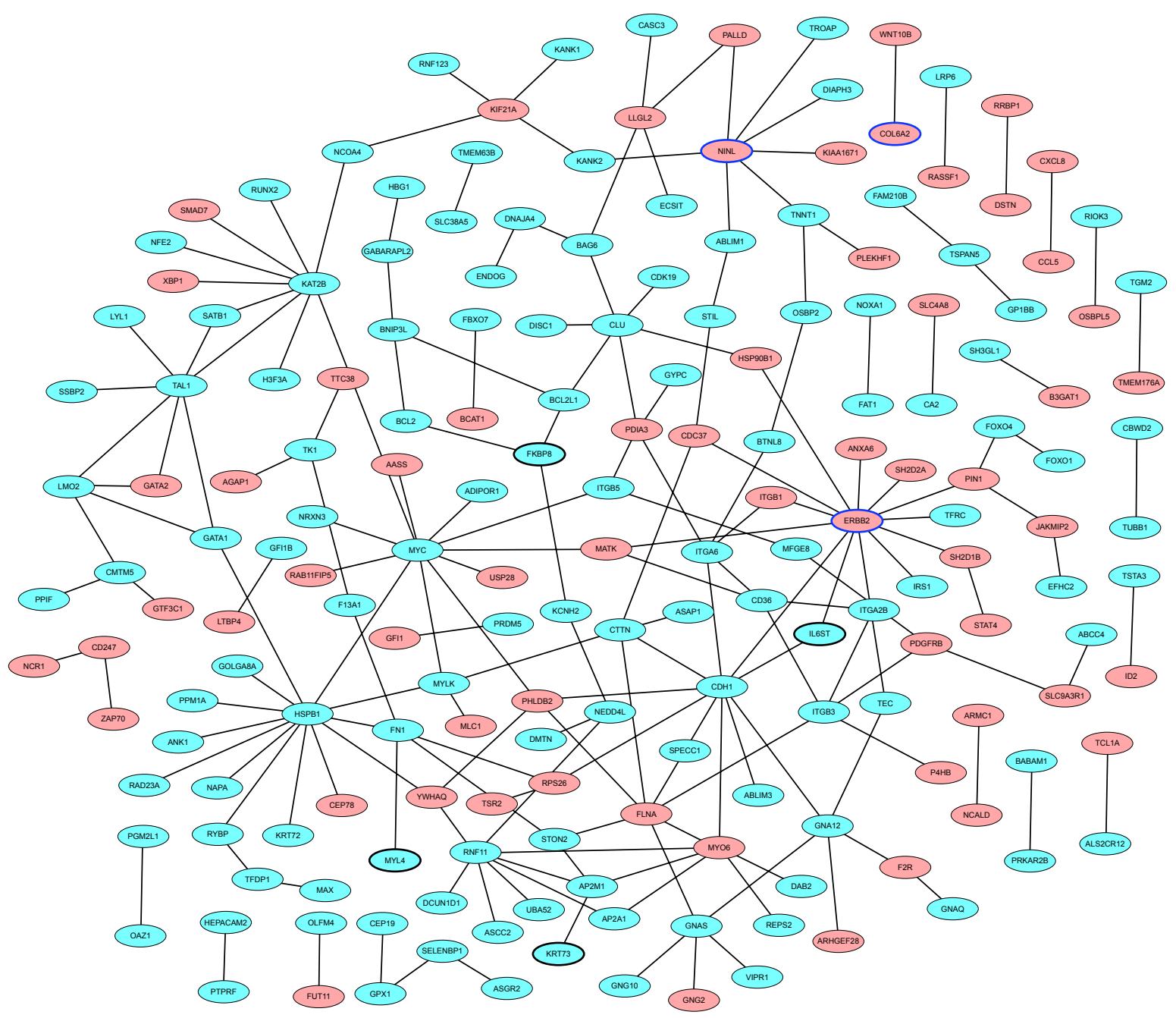

Figure 3 PD-specific PPI network.

Notes: The red and blue ellipses represent proteins encoded by up- and downregulated DEmRNAs between PD and normal control. Ellipses with black and blue border are DEmRNAs derived from top ten down- and upregulated DEmRNAs between PD and normal control.

Abbreviations: DEmRNAs, differentially expressed mRNAs; PD, Parkinson's disease; PPI, protein-protein interaction.

suggested a link between PD and HER2 polymorphism, and they indicated that different signals or potency of the kinase activities resulting from the Ala1170Pro allele of HER2 may be associated with vulnerability to stress on dopaminergic neurons in PD. ${ }^{25}$ In this present study, ERBB2 was a hub protein of PD-specific PPI network. Moreover, ERBB2 was a nearby targeted gene of LOC105372578, which suggested that LOC105372578 might be a potential regulator of PD by regulating $E R B B 2$.

$\mathrm{AD}$ is also a type of neurodegenerative disease. We identified three AD-regulated genes that were differentially expressed between PD and normal control as well.

ANK1 is a known susceptibility gene for type 2 diabetes which was recognized to own some similarities with AD. ${ }^{26}$ Aberrant methylation and expression of ANK1 were found in $\mathrm{AD} \cdot{ }^{27,28}$ Cortical-specific hypermethylation of ANK1 was robustly associated with AD-related neuropathology. ${ }^{28}$ Lunnon et al speculated that the brainexpressed ANK1 protein could be associated with pathology of $\mathrm{AD}$ by its function on compartmentalization of the plasma membrane. ${ }^{28}$

The triggering receptors expressed on myeloid (TREM) family is known to play a key role in modulating inflammation in the innate immune response. ${ }^{29}$ Both TREML1 and TREML4 were reported to be plausible risk genes of AD. ${ }^{30}$ Reliable expression of TREML1 was found in both cerebellum and temporal cortex of brain. ${ }^{30}$ Variant of TREM1 (rs6910730) was associated with increased AD pathology burden and increased rate of cognitive decline, independently. ${ }^{31}$ Moreover, TREML1 was reported to be involved with promoting vascular homeostasis and neuroinflammation that was speculated to be a potential mediator 
Table 3 Nearby targeted DEmRNAs of DElncRNAs between PD and normal controls

\begin{tabular}{|c|c|c|c|c|c|}
\hline \multicolumn{3}{|l|}{ DElncRNAs } & \multicolumn{3}{|c|}{ Nearby targeted DEmRNAs } \\
\hline Symbol & Start- 100 kb & End +100 kb & Symbol & Start & End \\
\hline CTD-220III8.I & 79891295 & 80183665 & SERINC5 & 80111226 & 80256082 \\
\hline EPHAI-ASI & 143253399 & | 43623449 & EPHAI & 143390813 & 143408892 \\
\hline EPHAI-ASI & 143253399 & 143623449 & FAMI3IB & 143353399 & 143382304 \\
\hline HCGII & 26386648 & 26627393 & BTN3AI & 26402237 & 26415216 \\
\hline HCGII & 26386648 & 26627393 & BTN3A2 & 26365158 & 26453415 \\
\hline KRT73-ASI & 52485588 & 52720133 & KRTI & 52674735 & 52680407 \\
\hline KRT73-ASI & 52485588 & 52720133 & KRT72 & 52585588 & 52602900 \\
\hline KRT73-ASI & 52485588 & 52720133 & KRT73 & 52585588 & 52620133 \\
\hline LEFI-ASI & 107947544 & 108276430 & LEFI & 108047544 & 108176430 \\
\hline LOCI01927012 & 147459993 & | 47882848 & JAKMIP2 & 147559993 & | 47782848 \\
\hline LOCI01927369 & 35997885 & 36256994 & CCL4 & 36097885 & 36156994 \\
\hline LOCI01928I00 & 10263768 & 10510146 & KLRDI & 10238384 & 10329607 \\
\hline LOCI01928I00 & 10263768 & 10510146 & KLRC4 & 10407384 & 10409757 \\
\hline LOCI01928I00 & 10263768 & 10510146 & KLRKI & 10372353 & 10390054 \\
\hline LOCIOI929866 & 45078476 & 45291638 & $\mathrm{PI} 3$ & 45174898 & 45176544 \\
\hline LOCI0I929866 & 45078476 & 45291638 & SLPI & 45230820 & 45290352 \\
\hline LOCI02724I04 & 39132519 & 39363406 & CX3CRI & 39263493 & 39281735 \\
\hline LOCI 05369772 & 52525675 & 52730770 & KRTI & 52674735 & 52680407 \\
\hline LOCI 05369772 & 52525675 & 52730770 & KRT72 & 52585588 & 52602900 \\
\hline LOCI 05369772 & 52525675 & 52730770 & KRT73 & 52585588 & 52620133 \\
\hline LOCI 05370556 & 70420816 & 70664742 & TTC9 & 70641786 & 70675360 \\
\hline LOCI0537I464 & I 59700480 & |599|6257 & FCRL6 & 159800480 & $1598 \mid 6257$ \\
\hline LOCI0537249I & 560703 & 796189 & SRXNI & 646614 & 658840 \\
\hline LOCI 05372578 & 24819978 & 25032985 & CST7 & 24949229 & 24959928 \\
\hline LOCI053727I6 & 62683185 & 62894281 & COL9A3 & $628|706|$ & 62937952 \\
\hline LOCI0537288I & $20726582 \mid$ & 207473252 & CD55 & $20732|47|$ & 207360966 \\
\hline LOCI 05373943 & 235394088 & 236231800 & AGAPI & 235494088 & 236131800 \\
\hline LOCI0537477I & 64290955 & 64525399 & LGALSL & 64454192 & 64461383 \\
\hline LOCI 05375056 & 41049096 & 41278568 & TREML4 & $4|22829|$ & 41239386 \\
\hline LOCI 05375056 & 41049096 & $4 \mid 278568$ & TREMLI & 41149096 & 41178568 \\
\hline LOCI 05375796 & $14345 \mid 962$ & 143663062 & TSTA3 & 143612617 & 143618043 \\
\hline LINC02084 & 27612180 & $278 \mid 4006$ & EOMES & 27715948 & $277227 \mid 5$ \\
\hline LOCI05377IIO & 58950175 & 61351474 & FHIT & 59050175 & 61251474 \\
\hline LOCI 05378678 & $4|32821|$ & $4|5649| 1$ & HIVEP3 & 41506364 & 42035925 \\
\hline LOCI0537870I & 47072216 & 47277080 & STIL & 47250138 & $473 \mid 4787$ \\
\hline LOCI0537870I & 47072216 & 47277080 & TALI & 47216289 & 47232389 \\
\hline LOCI 05379392 & 41553224 & 41996762 & ANKI & 41653224 & 41896762 \\
\hline SIRPG-ASI & $|529| 5 \mid$ & 1786516 & SIRPG & $|629| 5 \mid$ & 1686516 \\
\hline TMEM9B-ASI & 8847200 & 9076283 & AKIPI & 8911116 & 8933006 \\
\hline
\end{tabular}

Abbreviations: DElncRNAs, differentially expressed long noncoding RNAs; DEmRNAs, differentially expressed micro RNAs; PD, Parkinson's disease.

of neuronal protection and injury in AD and possibly other CNS diseases. ${ }^{30}$

To the best of our knowledge, we first found the downregulation of $A N K 1, T R E M L 1$, and TREML4 in PD compared with normal controls, which might involve with PD as well. Both TREML1 and TREML4 were nearby targeted genes of LOC105375056, and ANK1 was a nearby targeted gene of LOC105379392, which suggested that LOC105375056 and LOC105379392 and their interactions with TREML1/ TREML4 or ANK1 might implicate in PD.

In addition, several studies of the familial PD genes had emphasized the importance of RNA metabolism, particularly
mRNA translation, in the disease process. Bingwei Lu et al had indicated that several familial PD genes, including LRRK2, PINK1, Parkin, and eIF4G1, have been shown to interact with components of the translation initiation machinery or interact with modulators of the translation initiation process, such as miRNAs and the mTORC1 signaling pathway. ${ }^{32-34}$ In the future, the research on RNA metabolism in PD needs to be carried more.

According to the KEGG enrichment analysis, natural killer cell-mediated cytotoxicity was a significantly enriched pathway in PD. KLRD1 and killer cell lectin like receptor K1 (KLRK1) were the two DEmRNAs enriched in the pathway of natural 
A

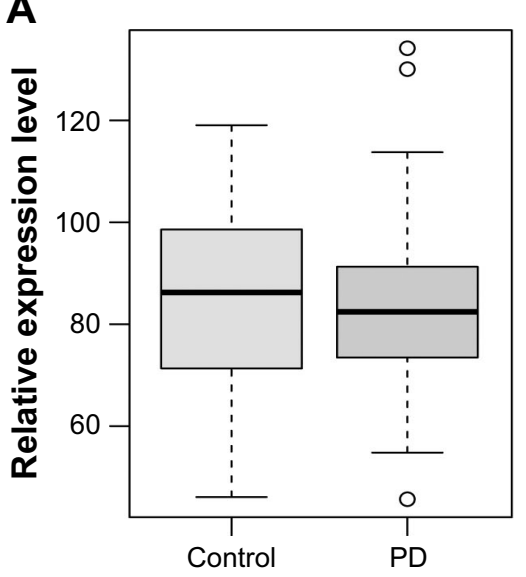

D

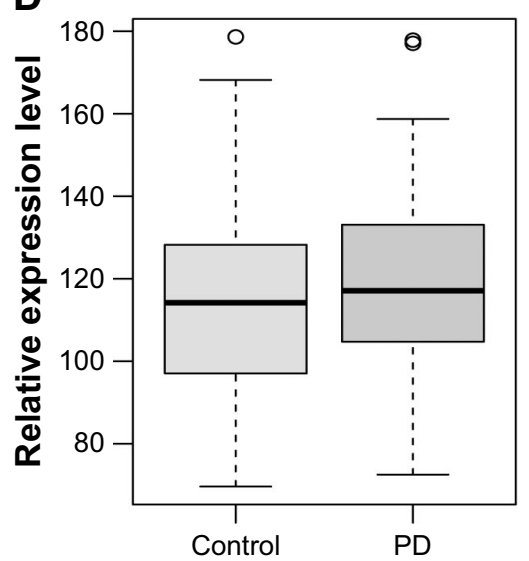

B

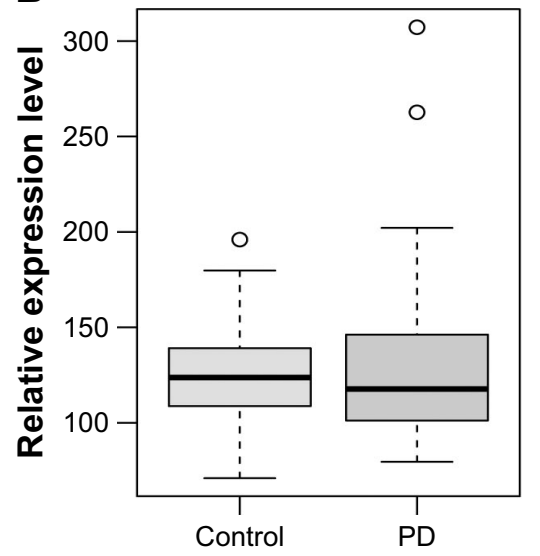

E

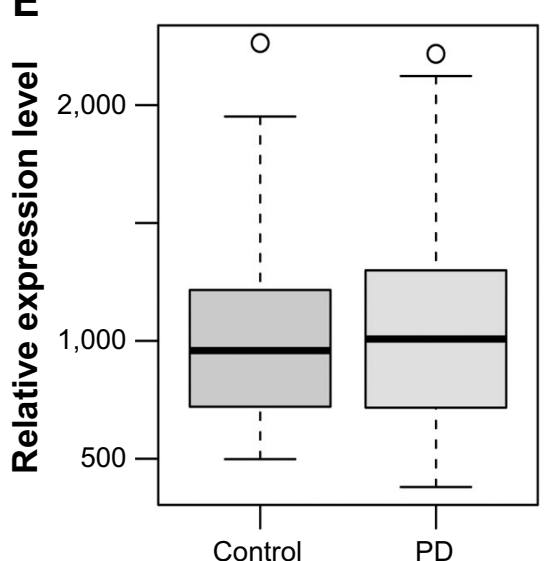

C

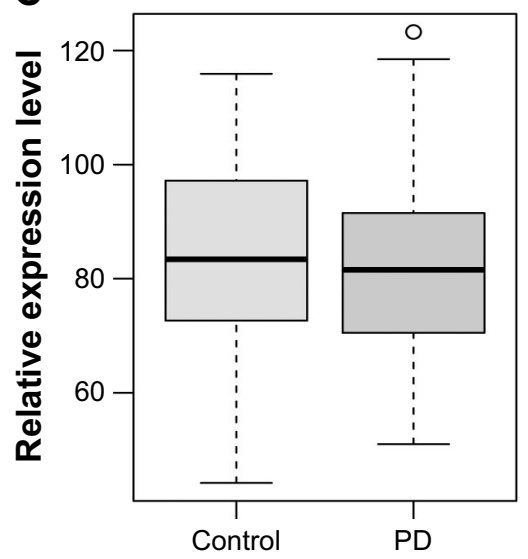

$\mathbf{F}$

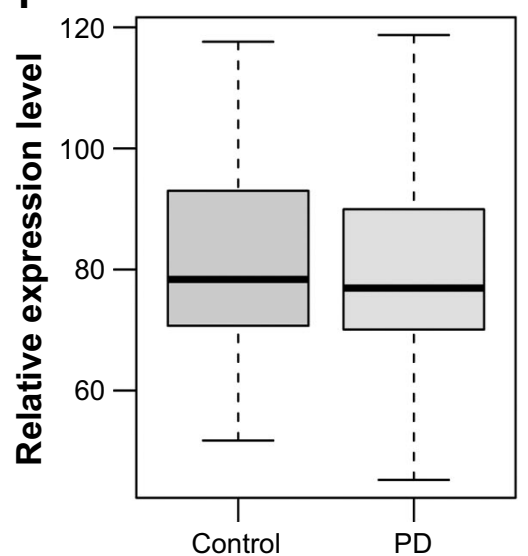

Figure 4 Validation of selected DEmRNAs in GSE57475

Notes: The $x$-axis shows PD and normal control groups and the $y$-axis shows relative expression levels. (A) TALI; (B) TREMLI; (C) TREML4; (D) ERBB2; (E) CX3CRI; (F) ANKI. The circles represent outliers.

Abbreviations: DEmRNAs, differentially expressed micro RNAs; PD, Parkinson's disease.

killer cell-mediated cytotoxicity, and KLRDI and KLRK1 were also the nearby targeted DEmRNAs of LOC101928100. These findings suggested the potential roles of LOC101928100 and its interactions with KLRK1/KLRD1 in PD.

\section{Conclusion}

Our data showed that an abundant of novel DElncRNAs may be associated with the pathology of PD. IncRNA interaction pairs of JHDM1D-AS1, LOC105378701-TAL1,
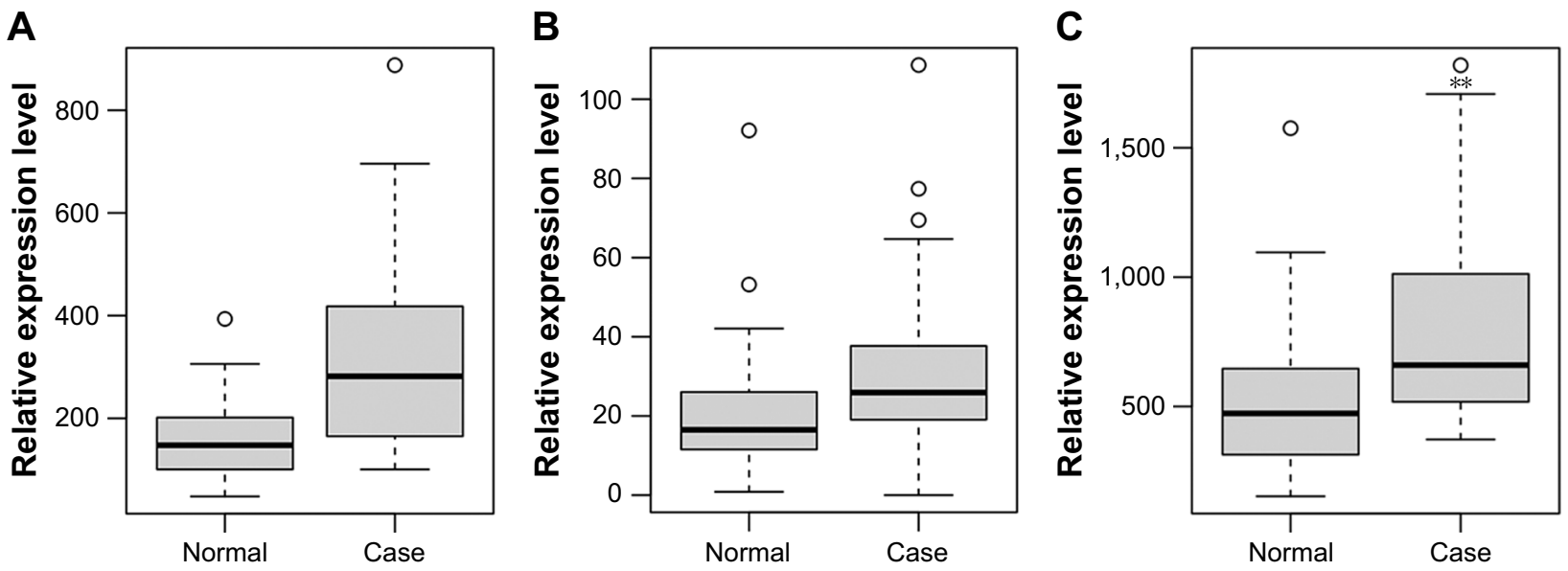

Figure 5 (Continued) 

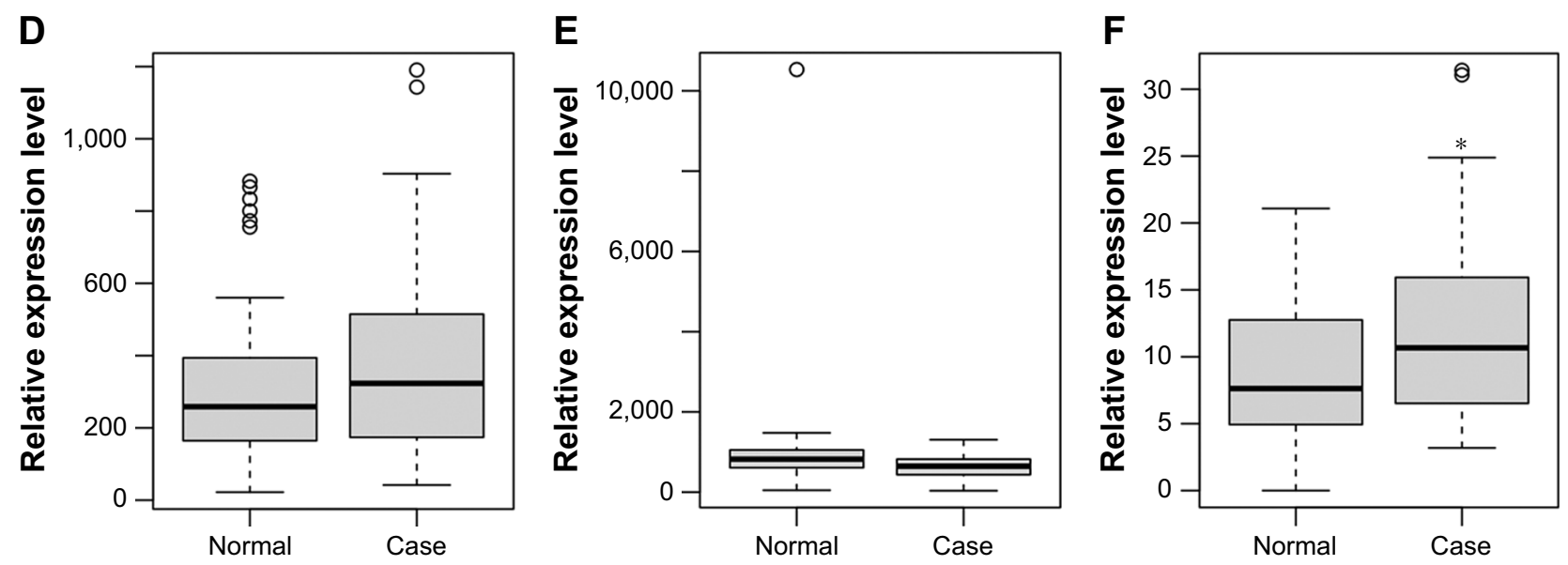

Figure 5 Validation of selected DEmRNAs in GSE687/9.

Notes: The $x$-axis shows PD and normal control groups and the $y$-axis shows relative expression levels. (A) TALI; (B) TREMLI; (C) ERBB2; (D) CX3CRI; (E) ANKI; (F) KLRDI. The circles represent outliers. $* P<0.05$ and $* * P<0.01$.

Abbreviations: DEmRNAs, differentially expressed micro RNAs; PD, Parkinson's disease.

\section{LOC102724104-CX3CR1, LOC105375056-TREML1/} TREML4, LOC105379392-ANK1, and LOC101928100KLRK1/KLRD1 might involve with PD. Their detailed roles in PD need to be clarified in our future work.

\section{Limitations}

The sample size for RNA sequencing in this study was small. Although the validation based on GSE57475 and GSE68719 suggested that our RNA-sequencing results were generally convincing, studies with larger sample size are needed to confirm this conclusion.

\section{Acknowledgments}

We thank Beijing Yangshen Bioinformatic Technology for assistance in high-throughput sequencing and data analysis. This study was supported by Nantong people's livelihood demonstration and promotion project in 2015 (MS32015033).

\section{Disclosure}

The authors report no conflicts of interest in this work.

\section{References}

1. Desai Bradaric B, Patel A, Schneider JA, Carvey PM, Hendey B. Evidence for angiogenesis in Parkinson's disease, incidental Lewy body disease, and progressive supranuclear palsy. J Neural Transm. 2012; 119(1):59-71.

2. Majidinia M, Mihanfar A, Rahbarghazi R, Nourazarian A, Bagca B, Avci ÇB. The roles of non-coding RNAs in Parkinson's disease. Mol Biol Rep. 2016;43(11):1193-1204.

3. Qureshi IA, Mehler MF. Emerging roles of non-coding RNAs in brain evolution, development, plasticity and disease. Nat Rev Neurosci. 2012; 13(8):528-541.
4. Cao X, Yeo G, Muotri AR, Kuwabara T, Gage FH. Noncoding RNAs in the mammalian central nervous system. Annu Rev Neurosci. 2006; 29:77-103.

5. Soreq L, Guffanti A, Salomonis N, et al. Long non-coding RNA and alternative splicing modulations in Parkinson's leukocytes identified by RNA sequencing. PLoS Comput Biol. 2014;10(3):e1003517.

6. Yan W, Chen ZY, Chen JQ, Chen HM. LncRNA NEAT1 promotes autophagy in MPTP-induced Parkinson's disease through stabilizing PINK1 protein. Biochem Biophys Res Commun. 2018;496(4): 1019-1024.

7. Chen Y, Lian YJ, Ma YQ, Wu CJ, Zheng YK, Xie NC. LncRNA SNHG1 promotes $\alpha$-synuclein aggregation and toxicity by targeting miR-15b-5p to activate SIAH1 in human neuroblastoma SH-SY5Y cells. Neurotoxicology. 2018;68:212-221.

8. Coupland KG, Kim WS, Halliday GM, Hallupp M, Dobson-Stone C, Kwok JB. Role of the long non-coding RNA MAPT-AS1 in regulation of microtubule associated protein tau (MAPT) expression in Parkinson's disease. PLoS One. 2016;11(6): 0157924.

9. Liu S, Cui B, Dai ZX, Shi PK, Wang ZH, Guo YY. Long non-coding RNA HOTAIR promotes Parkinson's disease induced by MPTP through up-regulating the expression of LRRK2. Curr Neurovasc Res. 2016;13(2):115-120.

10. Fan Y, Xiao S. Progression rate associated peripheral blood biomarkers of Parkinson's disease. J Mol Neurosci. 2018;65(3):312-318.

11. Chi J, Xie Q, Jia J, et al. Integrated analysis and identification of novel biomarkers in Parkinson's disease. Front Aging Neurosci. 2018; 10:178.

12. Postuma RB, Berg D, Stern M, et al. MDS clinical diagnostic criteria for Parkinson's disease. Mov Disord. 2015;30(12):1591-1601.

13. Kondo A, Nonaka A, Shimamura T, et al. Long noncoding RNA JHDM1D-AS1 promotes tumor growth by regulating angiogenesis in response to nutrient starvation. Mol Cell Biol. 2017;37(18):e00125-17.

14. Appel SH. Inflammation in Parkinson's disease: cause or consequence? Mov Disord. 2012;27(9):1075-1077.

15. Thome AD, Standaert DG, Harms AS. Fractalkine signaling regulates the inflammatory response in an $\alpha$-Synuclein model of Parkinson disease. PLoS One. 2015;10(10):e0140566.

16. Desai BS, Schneider JA, Li JL, Carvey PM, Hendey B. Evidence of angiogenic vessels in Alzheimer's disease. J Neural Transm. 2009; 116(5):587-597.

17. Ruiz-Orera J, Messeguer X, Subirana JA, Alba MM. Long non-coding RNAs as a source of new peptides. Elife. 2014;3:e03523. 
18. Galatro TF, Holtman IR, Lerario AM, et al. Transcriptomic analysis of purified human cortical microglia reveals age-associated changes. Nat Neurosci. 2017;20(8):1162-1171.

19. Wehrspaun CC, Haerty W, Ponting CP. Microglia recapitulate a hematopoietic master regulator network in the aging human frontal cortex. Neurobiol Aging. 2015;36(8):2443.e9-2443.e20.

20. Diao H, Li X, Hu S, Liu Y. Gene expression profiling combined with bioinformatics analysis identify biomarkers for Parkinson disease. PLoS One. 2012;7(12):e52319.

21. Hughes PM, Botham MS, Frentzel S, Mir A, Perry VH. Expression of fractalkine (CX3CL1) and its receptor, CX3CR1, during acute and chronic inflammation in the rodent CNS. Glia. 2002;37(4):314-327.

22. Ransohoff RM, Cardona AE. The myeloid cells of the central nervous system parenchyma. Nature. 2010;468(7321):253-262.

23. Cardona AE, Pioro EP, Sasse ME, et al. Control of microglial neurotoxicity by the fractalkine receptor. Nat Neurosci. 2006;9(7):917-924.

24. Sun S, Tang HM, Feng Y, et al. New evidences for fractalkine/CX3CL1 involved in substantia nigral microglial activation and behavioral changes in a rat model of Parkinson's disease. Neurobiol Aging. 2011;32(3):443-458.

25. Wang V, Chuang TC, Kao MC, Shan DE, Soong BW, Shieh TM. Polymorphic Ala-allele carriers at residue 1170 of HER2 associated with Parkinson's disease. J Neurol Sci. 2013;325(1-2):115-119.

26. Jayaraman A, Pike CJ. Alzheimer's disease and type 2 diabetes: multiple mechanisms contribute to interactions. Curr Diab Rep. 2014; 14(4):476.

27. De Jager PL, Srivastava G, Lunnon K, Burgess J, Schalkwyk LC. Alzheimer's disease: early alterations in brain DNA methylation at ANK1, BIN1, RHBDF2 and other loci. Nat Neurosci. 2014;17(9):1156-1163.

28. Lunnon K, Smith R, Hannon E, et al. Methylomic profiling implicates cortical deregulation of ANK1 in Alzheimer's disease. Nat Neurosci. 2014;17(9):1164-1170.

29. Ford JW, McVicar DW. TREM and TREM-like receptors in inflammation and disease. Curr Opin Immunol. 2009;21(1):38-46.

30. Carrasquillo MM, Allen M, Burgess JD, et al. A candidate regulatory variant at the TREM gene cluster associates with decreased Alzheimer's disease risk and increased TREML1 and TREM2 brain gene expression. Alzheimers Dement. 2017;13(6):663-673.

31. Replogle JM, Chan G, White CC, et al. A TREM1 variant alters the accumulation of Alzheimer-related amyloid pathology. Ann Neurol. 2015;77(3):469-477.
32. Lu B, Gehrke S, Wu Z. RNA metabolism in the pathogenesis of Parkinson's disease. Brain Res. 2014;1584:105-115.

33. Wu Z, Wang Y, Lim J. Ubiquitination of ABCE1 by NOT4 in response to mitochondrial damage links co-translational quality control to PINK1-directed mitophagy. Cell Metab. 2018;28(1):130.e7-144.e7.

34. Gehrke S, Wu Z, Klinkenberg M, et al. PINK1 and Parkin control localized translation of respiratory chain component mRNAs on mitochondria outer membrane. Cell Metab. 2015;21(1):95-108.

35. Langouet-Astrie C, Meinsen A, Grunwald E, Turner S, Enke R. RNA sequencing analysis of the developing chicken retina. Scientific Data. 2016;3:160117.

36. Martin M. Cutadapt removes adapter sequences from high-throughput sequencing reads. EMBnet $J$. 2011;17(1).

37. Kim D, Pertea G, Trapnell C, Pimentel H, Kelley R, Salzberg SL. TopHat2: accurate alignment of transcriptomes in the presence of insertions, deletions and gene fusions. Genome Biology. 2013;14(4):R36.

38. Church DM, Schneider VA, Graves T, et al. Modernizing Reference Genome Assemblies. Plos Biology. 2011;9(7):e1001091.

39. Trapnell C, Williams B, Pertea G, et al. Transcript assembly and quantification by RNA-Seq reveals unannotated transcripts and isoform switching during cell differentiation. Nature Biotechnology. 2010;28(5):511-515.

40. Love MI, Huber W, Anders S. Moderated estimation of fold change and dispersion for RNA-seq data with DESeq2. Genome Biology. 2014;15(12):550.

41. Tabasmadrid D, Nogalescadenas R, Pascualmontano A. GeneCodis3: a non-redundant and modular enrichment analysis tool for functional genomics. Nucleic Acids Research. 2012;40(Web Server issue):W478-W483.

42. Stark C, Breitkreutz B, Reguly T, Boucher L, Breitkreutz A, Tyers M. BioGRID: a general repository for interaction datasets. Nucleic Acids Research. 2006;34(Database issue):535-539.

43. Shannon P, Markiel A, Ozier O, et al. Cytoscape: a software environment for integrated models of biomolecular interaction networks Genome Research. 2003;13(11):2498.

44. Edgar R, Domrachev M, Lash A. Gene Expression Omnibus: NCBI gene expression and hybridization array data repository. Nucleic Acids Research. 2002;30(1):207-210.
Neuropsychiatric Disease and Treatment

\section{Publish your work in this journal}

Neuropsychiatric Disease and Treatment is an international, peerreviewed journal of clinical therapeutics and pharmacology focusing on concise rapid reporting of clinical or pre-clinical studies on a range of neuropsychiatric and neurological disorders. This journa is indexed on PubMed Central, the 'PsycINFO' database and CAS,

\section{Dovepress}

and is the official journal of The International Neuropsychiatric Association (INA). The manuscript management system is completely online and includes a very quick and fair peer-review system, which is all easy to use. Visit http://www.dovepress.com/testimonials.php to read real quotes from published authors. 